BMJ Open

Diabetes

Research

\& Care

\title{
Prevalence and major risk factors of type 2 diabetes mellitus among adult psychiatric inpatients from 2005 to 2018 in Beijing, China: a longitudinal observational study
}

\author{
Fude Yang, ${ }^{1}$ Qiuyue Ma, ${ }^{2}$ Jue Liu, ${ }^{2}$ Botao Ma, ${ }^{1}$ Moning Guo, ${ }^{3}$ Fangchao Liu, ${ }^{2}$ \\ Juan $\mathrm{Li}^{4}{ }^{4}$ Zhiren Wang, ${ }^{1}$ Min Liu (D) ${ }^{2}$
}

To cite: Yang $F$, Ma Q, Liu J, et al. Prevalence and major risk factors of type 2 diabetes mellitus among adult psychiatric inpatients from 2005 to 2018 in Beijing, China: a longitudinal observational study. BMJ Open Diab Res Care 2020;8:e000996. doi:10.1136/ bmjdrc-2019-000996

$\mathrm{FY}$ and $\mathrm{QM}$ contributed equally.

Received 20 0ctober 2019 Revised 14 February 2020 Accepted 15 February 2020

Check for updates

C A Author(s) (or their employer(s)) 2020. Re-use permitted under CC BY-NC. No commercial re-use. See rights and permissions. Published by BMJ.

For numbered affiliations see end of article.

Correspondence to Dr Min Liu; liumin@bjmu.edu.cn and Dr Fude Yang;

yangfd200@126.com

\section{ABSTRACT}

Objective We aim to investigate the prevalence, trends, and major risk factors of type 2 diabetes mellitus (T2DM) among adult psychiatric inpatients in Beijing, China. Research design and methods We did a longitudinal observational study using data from the Beijing Municipal Commission of Health and Family Planning Information Center, including 157570 adult psychiatric inpatients in 19 specialized psychiatric hospitals from 2005 to 2018 in Beijing. Data on demographic characteristics and antipsychotic medication use were obtained from electronic health records. Schizophrenia, T2DM, and comorbidities were defined according to the International Classification of Diseases, 10th revision codes of discharge diagnosis. The overall prevalence of T2DM in adult psychiatric inpatients was calculated, and the annual prevalence of T2DM was calculated and adjusted to the overall participant population. Univariate and multivariate logistic regression analyses were performed to obtain crude ORs and adjusted ORs (aORs) on the risk of T2DM in patients with different demographic characteristics, schizophrenia, antipsychotic medication use, and different comorbidities. Age-specific prevalence of T2DM under a stratification of schizophrenia or other psychiatric disorders was calculated in the subgroup analysis. Results Out of 157570 adult inpatients, 16939 had T2DM, with a prevalence of $10.75 \%(95 \% \mathrm{Cl} 10.60 \%$ to $10.90 \%)$. The prevalence was $11.63 \%(95 \% \mathrm{Cl} 11.37 \%$ to $11.88 \%$ ) among patients with schizophrenia and $10.17 \%$ (95\% Cl $9.98 \%$ to $10.37 \%$ ) among patients with other psychiatric disorders. During 2005-2018, the prevalence of T2DM in adult patients increased over the years, from an adjusted prevalence of $5.20 \%$ in 2005 , to $10.98 \%$ in $2010,12.50 \%$ in 2015 , and $12.71 \%$ in 2018 . Results from the multivariate analysis showed that increasing age, diagnosis of schizophrenia $(\mathrm{aOR}=1.23,95 \% \mathrm{Cl} 1.18$ to 1.29), and comorbidities of hypertension ( $\mathrm{aOR}=3.09,95 \%$ $\mathrm{Cl} 2.97$ to 3.22), lipid disorders ( $\mathrm{aOR}=1.95,95 \% \mathrm{Cl} 1.88$ to 2.04), and fatty liver (aOR=1.93, 95\% $\mathrm{Cl} 1.84$ to 2.03) were major risk factors of T2DM in adult psychiatric inpatients. Conclusions The prevalence of T2DM was high among adult psychiatric inpatients in Beijing, China. Elderly patients, those with schizophrenia, and those with hypertension, lipid disorders, and fatty liver had higher

\section{Significance of this study}

What is already known about this subject?

- The prevalence of diabetes mellitus is often reported to be higher in psychiatric inpatients than in the general population.

- However, large studies on diabetes prevalence among psychiatric inpatients in China are scant, and the trends in the prevalence of type 2 diabetes mellitus (T2DM) and its associated risk factors in Chinese adult psychiatric inpatients remain unknown.

What are the new findings?

- The prevalence of T2DM was $10.75 \%$ among adult psychiatric inpatients in Beijing during 2005-2018.

- The prevalence of T2DM in adult patients increased over time, from an adjusted prevalence of $5.20 \%$ in 2005 , to $10.98 \%$ in $2010,12.50 \%$ in 2015 , and $12.71 \%$ in 2018.

- Multivariate logistic regression analysis showed that advanced age, schizophrenia diagnosis, and comorbidities of hypertension, lipid disorders, and fatty liver were major risk factors for T2DM in adult psychiatric inpatients.

How might these results change the focus of research or clinical practice?

- The prevalence of T2DM was high among adult psychiatric inpatients in Beijing, China.

- Elderly psychiatric inpatients and those with schizophrenia, hypertension, lipid disorders, and fatty liver should be given monitoring, prevention and treatment for T2DM.

prevalence of T2DM. Prevention and treatment of T2DM are of utmost relevance in hospitalized psychiatric patients.

\section{INTRODUCTION}

Diabetes mellitus (DM) is a global public health crisis that threatens the economies of all nations, particularly those of developing countries. ${ }^{1}$ Type 2 diabetes mellitus (T2DM), 
resulting from impaired insulin secretion, insulin resistance, or a combination of both conditions, is far more common than other types of DM; T2DM accounts for more than $90 \%$ of all DM cases. ${ }^{2}$ According to an estimate by the International Diabetes Federation, 425 million adults aged 20-79 years worldwide had T2DM in 2017, and the areas that were particularly affected by DM were China and India. ${ }^{3}$ Rapid socioeconomic development and considerable changes in dietary patterns, behavior, and lifestyle have contributed to the rapidly increasing risk of T2DM. ${ }^{4}$ In China, the prevalence of DM has grown rapidly in the past decade, increasing from $2.6 \%$ in 2002 to $10.9 \%$ in $2013 .{ }^{45}$

DM imposes a substantial physical and psychological burden on patients, resulting in a decline in health status and quality of life. ${ }^{2}$ The prevalence of DM is often reported to be higher in psychiatric inpatients than in the general population. ${ }^{6}$ Furthermore, the prevalence of DM is generally reported to be higher in people with psychotic disorders than in those with other types of mental illnesses. ${ }^{7}$ Patients with serious mental illness (SMI), such as schizophrenia, demonstrate 1.5-2fold prevalence of T2DM and have poorer long-term outcomes, including increased tendency for relapse of mental illness and increased mortality secondary to an increased risk of cardiovascular disease. ${ }^{68}$

Age, obesity, hypertension, hyperlipidemia, and sedentary lifestyle are well-documented risk factors for DM, and many of these risk factors are more common in people with psychotic disorders than in the general population, which is partly explained by adverse health behaviors. ${ }^{7}$ Moreover, patients with mental illness exhibit unique risk factors for DM, such as the use of antipsychotic medications. ${ }^{79}$ Adverse metabolic effects of second-generation antipsychotics are well documented, including obesity, $\mathrm{DM}$, hypertension, and so on. ${ }^{10} \mathrm{~A}$ large-scale metaanalysis reported that approximately 1 in 10 individuals with SMI (11.3\%) had T2DM, and the relative risk (RR) of T2DM in multiepisode persons with SMIs was almost double $(R R=1.85)$ that in matched individuals from the general population. ${ }^{9}$

Randomized controlled trials have conclusively demonstrated that intensive lifestyle interventions and medications effectively delay or prevent the development of T2DM in high-risk individuals. ${ }^{2}$ Therefore, knowledge of factors associated with a high risk of DM is crucial for specialized psychiatric hospitals, and can help identify individuals with the greatest need for intensive monitoring and intervention, ${ }^{9}$ and improve clinical outcomes as well as quality of life in these disadvantaged populations. ${ }^{11}$

Although the prevalence and risk factors for DM in psychiatric patients are well documented, data on the prevalence and risk factors for T2DM among Chinese psychiatric inpatients are scarce. Reported prevalence estimates of DM among psychiatric inpatients range from $12.5 \%$ to $25.7 \%$ and are often based on small studies or studies on patients with a specific mental illness. ${ }^{12} 13$ We analyzed electronic health records (EHR) data of 157570 inpatients aged at least 18 years during 2005-2018 from all 19 specialized psychiatric hospitals in Beijing to estimate the prevalence of, trends in, and major risk factors for T2DM in adult psychiatric inpatients. The objective was to identify high-risk individuals and detect and provide treatment for abnormal glucose metabolism among them.

\section{METHODS}

\section{Study design and study population}

This was a longitudinal observational study. We used EHR data obtained from the Beijing Municipal Commission of Health and Family Planning Information Center, which covered all 19 specialized psychiatric hospitals in Beijing, China. Among these hospitals, four were third-level psychiatric hospitals, with a bed capacity of over 500 , and 15 hospitals were second-level psychiatric hospitals, with a bed capacity below 500 . The study population included all inpatients who were discharged from the aforementioned 19 psychiatric hospitals between January 1, 2005 and December 31, 2018. The total number of inpatients was 197990 . After excluding 11874 inpatients aged $<18$ years as well as 28546 inpatients because they were readmitted within the same calendar year, 157570 adult inpatients were included in the final analysis.

\section{Disease diagnosis and ICD-10 codes}

Information on diseases was obtained from hospital discharge diagnoses, including all primary and secondary diagnoses, which were coded by physicians according to the International Classification of Diseases, 10th revision (ICD-10). ${ }^{14}$

The ICD-10 code for schizophrenia is F20, which includes paranoid schizophrenia, hebephrenic schizophrenia, catatonic schizophrenia, undifferentiated schizophrenia, postschizophrenic depression, residual schizophrenia, simple schizophrenia, other schizophrenia, and schizophrenia, unspecified.

In this study, T2DM included ICD-10 codes E11 (noninsulin-dependent DM) and E14 (unspecified DM).

Comorbidities were also identified using ICD-10 codes: hypertension: I10-I15 (including essential hypertension, hypertensive heart disease, hypertensive renal disease, hypertensive heart and renal disease, and secondary hypertension); lipid disorders: E78 (disorders of lipoprotein metabolism and other lipidemias); and fatty liver: K76.0.

Furthermore, antipsychotic medication use was defined as medical records listing a prescription for antipsychotic medication.

\section{Outcome variable}

We identified patients with T2DM using ICD-10 codes in all primary and secondary hospital discharge diagnoses. 


\section{Covariates}

The following demographic characteristics which may be associated with DM were used as adjustment variables: gender (male, female), age (18-29, 30-39, 40-49, 50-59, $60-69,70-79$, and $\geq 80$ years), ethnic origin (Han, and other ethnicity), marital status (never married, married, divorced, widowed, and unknown), payment (new rural cooperative medical scheme (NCMS), urban employee basic medical insurance (UEBMI), urban resident basic medical insurance (URBMI), other insurance, and outof-pocket expenditure), and hospital level (third-level and second-level hospitals). Diagnosis of schizophrenia or other psychiatric disorders (including bipolar affective disorder, recurrent depressive disorder, depressive episode, mental and behavioral disorders due to use of alcohol, acute and transient psychotic disorders, persistent delusional disorders, manic episode, obsessivecompulsive disorder, and schizoaffective disorders), antipsychotic medication use, and comorbidities of hypertension, lipid disorders, and fatty liver were also considered adjustment variables.

\section{Statistical analyses}

Proportions were used to describe data related to demographic characteristics, schizophrenia diagnosis, antipsychotic medication use, and comorbidities. T2DM prevalence was calculated using percentages and 95\% CIs. The annual prevalence rates of T2DM from 2005 to 2018 were evaluated for all inpatients and separately for men and women using percentages and 95\% CIs. Ageadjusted T2DM prevalence was calculated using direct standardization method, and all participants included in this study were used as a reference population. We used $\chi^{2}$ test to compare the prevalence among inpatients with different characteristics. Univariate and multivariate logistic regression methods were used to analyze factors associated with T2DM. Crude ORs and adjusted ORs (aORs) with 95\% CIs were calculated. The prevalence of T2DM in different age groups after stratification by schizophrenia diagnosis was also calculated. All analyses were conducted using SPSS Statistics for Windows, V.19.0.

\section{Patient and public involvement}

Patients and the public were not involved in the design and conduct of the study. But the study findings will be disseminated through multiple channels including publication, meetings, conferences and social media.

\section{RESULTS}

We enrolled 157570 inpatients aged at least 18 years from all 19 specialized psychiatric hospitals in Beijing during 2005-2018. The mean age of all inpatients was $43.53 \pm 17.16$ years. The demographic characteristics of inpatients were as follows: $78333(49.71 \%)$ were men, $71552(45.40 \%)$ were aged between 18 and 39 years, 148 $320(94.13 \%)$ were of Han ethnicity, $79583(50.51 \%)$ were married, $61713(39.17 \%)$ had UEBMI, and 126
$884(80.53 \%)$ were discharged from third-level hospitals. Among all inpatients, $62533(39.69 \%)$ were diagnosed with schizophrenia and 51778 (32.86\%) had prescriptions for antipsychotic medications (table 1).

Among the 157570 inpatients, 16939 were diagnosed with T2DM, with a prevalence of $10.75 \%$ (95\% CI $10.60 \%$ to $10.90 \%)$. We calculated the prevalence of T2DM using the year in which the inpatients were admitted to the hospital. Logistic regression results showed that the prevalence of T2DM of all inpatients increased with time from 2005 to 2018. The adjusted prevalence of T2DM increased from $5.20 \%$ in 2005 to $12.71 \%$ in 2018 . In male inpatients, the adjusted prevalence of T2DM increased from $5.56 \%$ in 2005 to $13.99 \%$ in 2018 . In female inpatients, the adjusted prevalence of T2DM increased from $4.93 \%$ in 2005 to $11.62 \%$ in 2018 (table 2, figure 1).

The prevalence of T2DM was slightly higher in women than in men $(11.03 \%$ vs $10.47 \%, \mathrm{p}<0.001)$. The prevalence of T2DM increased with age, with prevalence of $0.84 \%-3.54 \%$ in inpatients aged 18-39 years, $9.15 \%-$ $24.42 \%$ in inpatients aged 40-69 years, and more than $28 \%$ in inpatients aged $\geq 70$ years. The prevalence of T2DM was higher in inpatients of Han ethnicity than in those of other ethnicities $(10.84 \%$ vs $9.37 \%, \mathrm{p}<0.001)$. Divorced and widowed inpatients had higher T2DM prevalence $(18.73 \%$ and $18.60 \%$ ), and inpatients who were never married had the lowest T2DM prevalence $(5.12 \%)$. Inpatients with UEBMI and URBMI had higher T2DM prevalence (17.93\% and $16.45 \%)$, and those with NCMS had the lowest T2DM prevalence (4.06\%). Inpatients from second-level hospitals had higher T2DM prevalence than those from third-level hospitals $(21.65 \%$ vs $8.12 \%, \mathrm{p}<0.001)$.

The prevalence of T2DM was significantly higher in inpatients with schizophrenia than in those with other psychiatric disorders $(11.63 \%$ vs $10.17 \%, \mathrm{p}<0.001)$. Inpatients with antipsychotic prescription exhibited significantly higher T2DM prevalence than those without antipsychotic prescription $(12.14 \%$ vs $10.07 \%, \mathrm{p}<0.001)$. Inpatients with hypertension exhibited significantly higher T2DM prevalence than those without hypertension $(34.88 \%$ vs $6.36 \%, \mathrm{p}<0.001)$. Inpatients with lipid disorders had significantly higher T2DM prevalence than those without lipid disorders $(26.40 \%$ vs $8.16 \%, \mathrm{p}<0.001)$. Inpatients with fatty liver had significantly higher T2DM prevalence than those without fatty liver $(24.93 \%$ vs $9.14 \%, \mathrm{p}<0.001$; table 1 ).

The results of multivariate logistic regression analysis showed that female sex and non-Han ethnicity were not associated with a high risk of T2DM; however, age, marital status, payment, and hospital level were associated with the prevalence of T2DM. Compared with inpatients in the 18-29 years age group, the aORs were 6.51 (95\% CI 5.78 to 7.33 ) in the $40-49$ years age group and 14.15 (95\% CI 12.47 to 16.06$)$ in the $70-79$ years age group. The aORs were 1.07 (95\% CI 1.01 to 1.12$)$ among inpatients who were married and 1.08 (95\% CI 1.01 to 1.16) among inpatients who were widowed compared 
Table 1 Prevalence of T2DM in adult psychiatric inpatients during 2005-2018, Beijing, China

\begin{tabular}{|c|c|c|c|c|c|c|}
\hline & $\mathbf{n}$ & $\%$ & T2DM (n) & $\begin{array}{l}\text { Prevalence of T2DM, } \\
\%(95 \% \mathrm{Cl})\end{array}$ & cOR $(95 \% \mathrm{Cl})$ & aOR $(95 \% \mathrm{Cl})$ \\
\hline All inpatients & 157570 & 100.00 & 16939 & 10.75 (10.60 to 10.90$)$ & - & - \\
\hline \multicolumn{7}{|l|}{ Gender } \\
\hline Male & 78333 & 49.71 & 8201 & $10.47(10.26$ to 10.68$)$ & 1.00 & 1.00 \\
\hline Female & 79237 & 50.29 & 8738 & 11.03 (10.81 to 11.25$)$ & 1.06 (1.03 to 1.09$)$ & 1.00 (0.97 to 1.04$)$ \\
\hline \multicolumn{7}{|l|}{ Age group, years } \\
\hline $18-29$ & 42063 & 26.69 & 355 & 0.84 (0.76 to 0.93$)$ & 1.00 & 1.00 \\
\hline 30-39 & 29489 & 18.71 & 1043 & 3.54 (3.33 to 3.75$)$ & 4.31 (3.82 to 4.86$)$ & $3.10(2.74$ to 3.51$)$ \\
\hline $40-49$ & 29160 & 18.51 & 2669 & 9.15 (8.82 to 9.48 ) & 11.84 (10.58 to 13.24$)$ & 6.51 (5.78 to 7.33 ) \\
\hline $50-59$ & 27244 & 17.29 & 4966 & 18.23 (17.77 to 18.69$)$ & 26.19 (23.49 to 29.20$)$ & 10.84 (9.64 to 12.19$)$ \\
\hline $60-69$ & 16150 & 10.25 & 3944 & 24.42 (23.76 to 25.08$)$ & 37.96 (33.99 to 42.40$)$ & $\begin{array}{l}12.72(11.28 \text { to } \\
14.35)\end{array}$ \\
\hline $70-79$ & 8980 & 5.70 & 2601 & 28.96 (28.03 to 29.90$)$ & 47.90 (42.74 to 53.69$)$ & $\begin{array}{l}14.15(12.47 \text { to } \\
16.06)\end{array}$ \\
\hline$\geq 80$ & 4484 & 2.85 & 1361 & 30.35 (29.01 to 31.70$)$ & 51.20 (45.31 to 57.86$)$ & $\begin{array}{l}12.23 \text { (10.66 to } \\
14.05)\end{array}$ \\
\hline \multicolumn{7}{|l|}{ Ethnic origin } \\
\hline Han & 148320 & 94.13 & 16072 & 10.84 (10.68 to 10.99$)$ & 1.00 & 1.00 \\
\hline Others & 9250 & 5.87 & 867 & 9.37 (8.78 to 9.97 ) & 0.85 (0.79 to 0.91$)$ & 1.04 (0.97 to 1.13$)$ \\
\hline \multicolumn{7}{|l|}{ Marital status } \\
\hline Never married & 59057 & 37.48 & 3022 & 5.12 (4.94 to 5.29$)$ & 1.00 & 1.00 \\
\hline Married & 79583 & 50.51 & 10389 & 13.05 (12.82 to 13.29$)$ & 2.78 (2.67 to 2.90$)$ & 1.07 (1.01 to 1.12 ) \\
\hline Divorced & 7980 & 5.06 & 1495 & 18.73 (17.88 to 19.59$)$ & 4.27 (4.00 to 4.57 ) & 1.07 (0.99 to 1.16$)$ \\
\hline Widowed & 10841 & 6.88 & 2016 & 18.60 (17.86 to 19.33$)$ & 4.24 (3.99 to 4.50$)$ & 1.08 (1.01 to 1.16$)$ \\
\hline Unknown & 109 & 0.07 & 17 & 15.60 (8.68 to 22.52$)$ & 3.43 (2.04 to 5.76 ) & 1.00 (0.57 to 1.74$)$ \\
\hline \multicolumn{7}{|l|}{ Payment } \\
\hline NCMS & 26159 & 16.60 & 1063 & 4.06 (3.82 to 4.30$)$ & 1.00 & 1.00 \\
\hline UEBMI & 61713 & 39.17 & 11067 & 17.93 (17.63 to 18.24$)$ & 5.16 (4.84 to 5.50$)$ & 1.94 (1.81 to 2.08$)$ \\
\hline URBMI & 6998 & 4.44 & 1151 & 16.45 (15.58 to 17.32$)$ & 4.65 (4.26 to 5.08$)$ & 1.61 (1.46 to 1.78$)$ \\
\hline Other insurance & 24211 & 15.37 & 1937 & 8.00 (7.66 to 8.34$)$ & 2.05 (1.90 to 2.22 ) & 1.38 (1.27 to 1.50$)$ \\
\hline OOP & 38489 & 24.43 & 1721 & 4.47 (4.26 to 4.68$)$ & 1.11 (1.02 to 1.19$)$ & 1.06 (0.98 to 1.15$)$ \\
\hline \multicolumn{7}{|l|}{ Hospital level } \\
\hline Third-level & 126884 & 80.53 & 10297 & 8.12 (7.97 to 8.27$)$ & 1.00 & 1.00 \\
\hline Second-level & 30686 & 19.47 & 6642 & 21.65 (21.18 to 22.11$)$ & 3.13 (3.02 to 3.24$)$ & 1.55 (1.48 to 1.61$)$ \\
\hline \multicolumn{7}{|l|}{ Schizophrenia } \\
\hline No & 95037 & 60.31 & 9669 & 10.17 (9.98 to 10.37$)$ & 1.00 & 1.00 \\
\hline Yes & 62533 & 39.69 & 7270 & 11.63 (11.37 to 11.88 ) & $1.16(1.12$ to 1.20$)$ & 1.23 (1.18 to 1.29$)$ \\
\hline \multicolumn{7}{|l|}{ Antipsychotics } \\
\hline No & 105792 & 67.14 & 10655 & 10.07 (9.89 to 10.25$)$ & 1.00 & 1.00 \\
\hline Yes & 51778 & 32.86 & 6284 & 12.14 (11.86 to 12.42$)$ & 1.23 (1.19 to 1.27$)$ & 1.02 (0.98 to 1.06$)$ \\
\hline \multicolumn{7}{|l|}{ Hypertension } \\
\hline No & 133336 & 84.62 & 8485 & 6.36 (6.23 to 6.49$)$ & 1.00 & 1.00 \\
\hline Yes & 24234 & 15.38 & 8454 & 34.88 (34.28 to 35.48$)$ & 7.88 (7.62 to 8.16$)$ & 3.09 (2.97 to 3.22$)$ \\
\hline \multicolumn{7}{|l|}{ Lipid disorders } \\
\hline No & 135203 & 85.81 & 11034 & 8.16 (8.02 to 8.31$)$ & 1.00 & 1.00 \\
\hline Yes & 22367 & 14.19 & 5905 & 26.40 (25.82 to 26.98$)$ & 4.04 (3.90 to 4.18$)$ & 1.95 (1.88 to 2.04$)$ \\
\hline
\end{tabular}




\begin{tabular}{|c|c|c|c|c|c|c|}
\hline & $\mathbf{n}$ & $\%$ & T2DM (n) & $\begin{array}{l}\text { Prevalence of T2DM, } \\
\%(95 \% \mathrm{CI})\end{array}$ & cOR $(95 \% \mathrm{Cl})$ & aOR $(95 \% \mathrm{Cl})$ \\
\hline \multicolumn{7}{|c|}{ Fatty liver } \\
\hline No & 141513 & 89.81 & 12936 & 9.14 (8.99 to 9.29$)$ & 1.00 & 1.00 \\
\hline Yes & 16057 & 10.19 & 4003 & 24.93 (24.26 to 25.60$)$ & 3.30 (3.17 to 3.44$)$ & 1.93 (1.84 to 2.03$)$ \\
\hline
\end{tabular}

aOR, adjusted OR; cOR, crude OR; NCMS, new rural cooperative medical scheme; OOP, out-of-pocket; T2DM, type 2 diabetes mellitus; UEBMI, urban employee basic medical insurance; URBMI, urban resident basic medical insurance.

with those who had never been married. Compared with inpatients with NCMS payment, the aORs were 1.94 (95\% CI 1.81 to 2.08) among those with UEBMI payment and 1.61 (95\% CI 1.46 to 1.78 ) among those with URBMI payment. The aORs among inpatients from second-level hospitals were 1.55 (95\% CI 1.48 to 1.61) compared with those from third-level hospitals.

In the multivariate logistic models for T2DM, the aOR of inpatients with schizophrenia was 1.23 (95\% CI 1.18 to 1.29) compared with inpatients with other psychiatric disorders. The aORs of inpatients with hypertension, lipid disorders, and fatty liver were 3.09 (95\% CI 2.97 to 3.22 ), 1.95 (95\% CI 1.88 to 2.04 ), and 1.93 (95\% CI 1.84 to 2.03 ), respectively. After adjustment for all covariates, the aOR of inpatients with antipsychotic medication prescription was 1.02 (95\% CI 0.98 to 1.06) compared with those without antipsychotic medication prescription (table 1).

We analyzed the prevalence of T2DM stratified by schizophrenia and other psychiatric disorders. The prevalence of T2DM increased with age in inpatients with schizophrenia and in those with other psychiatric disorders. In each age group, the prevalence of T2DM was higher in patients with schizophrenia than in those with other psychiatric disorders. The prevalence of T2DM was approximately two times higher among patients with schizophrenia in the 18-29 and 30-39 years age groups than among those in the other age groups (figure 2).

\section{DISCUSSION}

Individuals with SMI, including schizophrenia, bipolar disorder, schizoaffective disorder, and major depressive disorder, are prone to multiple physical health problems. ${ }^{11}$ Results from a meta-analysis showed that approximately 1 in 10 individuals with SMI $(11.3 \%)$ had T2DM. ${ }^{9}$ In this study, we found that the prevalence of T2DM among adult psychiatric inpatients was $10.75 \%$ in Beijing, which is lower than that reported in the aforementioned meta-analysis. Zou and colleagues ${ }^{15}$ used Chinese and US data sets to confirm the novel diabetes subgroups proposed by Ahlqvist and colleagues, ${ }^{16}$ which used six variables to identify five exclusive diabetes subgroups. The novel groups provide a step toward a more precise diabetes stratification. Due to the limited data in our study, we could not identify diabetes by using the novel subgroups; such stratification can be applied in future studies.

The most commonly diagnosed specific chronic disease among schizophrenia patients is diabetes, which was reported to affect $12.5 \%$ of women and $11.0 \%$ of men in a Swedish national cohort study followed up for 7 years. ${ }^{17}$ Our results are consistent with the Swedish study, with a T2DM prevalence of $11.03 \%$ in female patients with schizophrenia and of $10.47 \%$ in male patients with schizophrenia. In our study, the overall prevalence of T2DM in patients with schizophrenia was $11.63 \%$, which is lower than the previous estimate of $12.50 \%$ in patients aged 22-76 years with schizophrenia in a Chinese hospital. ${ }^{12}$ Since 2005, the T2DM prevalence among adult psychiatric inpatients has increased with time; the highest prevalence rate among women was observed in 2015 and that among men was observed in 2018. Although the reason for T2DM prevalence increasing over the years might be the improvement in assessment of diabetes over the years, the diabetes prevalence has also increased in the general population from $2.6 \%$ in 2002 to $10.9 \%$ in $2013 .{ }^{45}$

The prevalence of T2DM in psychiatric inpatients increased with age, which is consistent with the results of a study conducted on patients with SMI in the UK. ${ }^{18}$ The prevalence of diabetes in inpatients aged 60-69 years $(24.42 \%)$ was only slightly lower than that (25.7\%) in hospitalized elderly patients with schizophrenia in Shanghai. ${ }^{13}$ Compared with the overall DM prevalence in the general Chinese adult population $(10.9 \%),{ }^{5}$ that of psychiatric inpatients was slightly lower (10.75\%). However, in the $<40$ years age group, psychiatric inpatients had lower diabetes prevalence $(0.84 \%-3.54 \%)$ than the general adult population (5.9\%). In the 40-59 years age group $(9.15 \%-18.23 \%)$ and $\geq 60$ years age group $(24.42 \%-30.35 \%)$, psychiatric inpatients had higher diabetes prevalence than general adults $(12.9 \%$ and $20.2 \%$, respectively) ${ }^{5}$ In the general population, age was an important risk factor of T2DM; hence, it should not be considered a risk factor specific to adult psychiatric inpatients.

Multivariate analysis revealed that gender was not related to the prevalence of T2DM, which is consistent with the findings of other research. ${ }^{19}$ Wang and colleagues $^{5}$ estimated the prevalence of diabetes in the Chinese adult population, and they found that the crude prevalence of diabetes was lower among major minority 


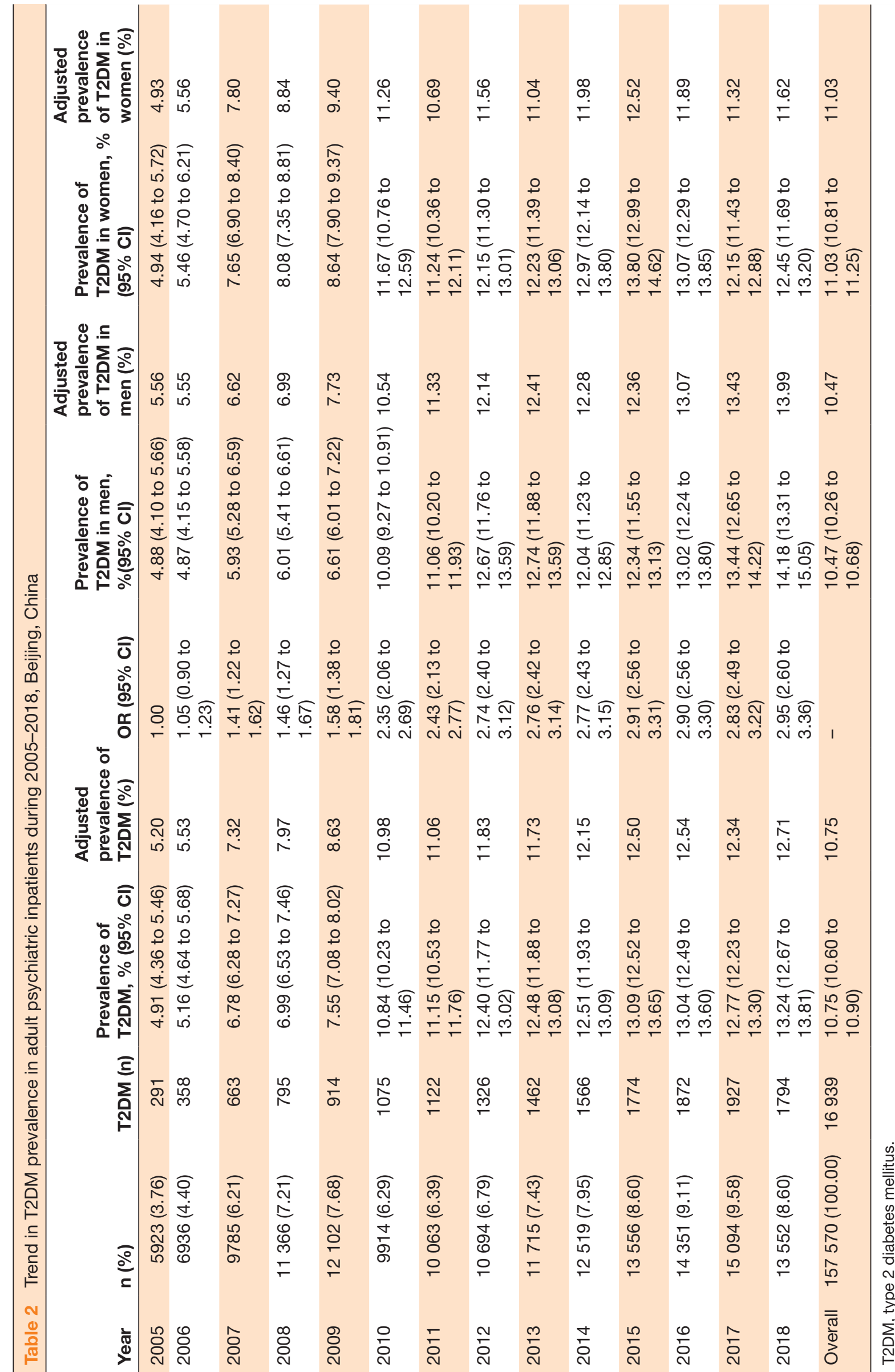



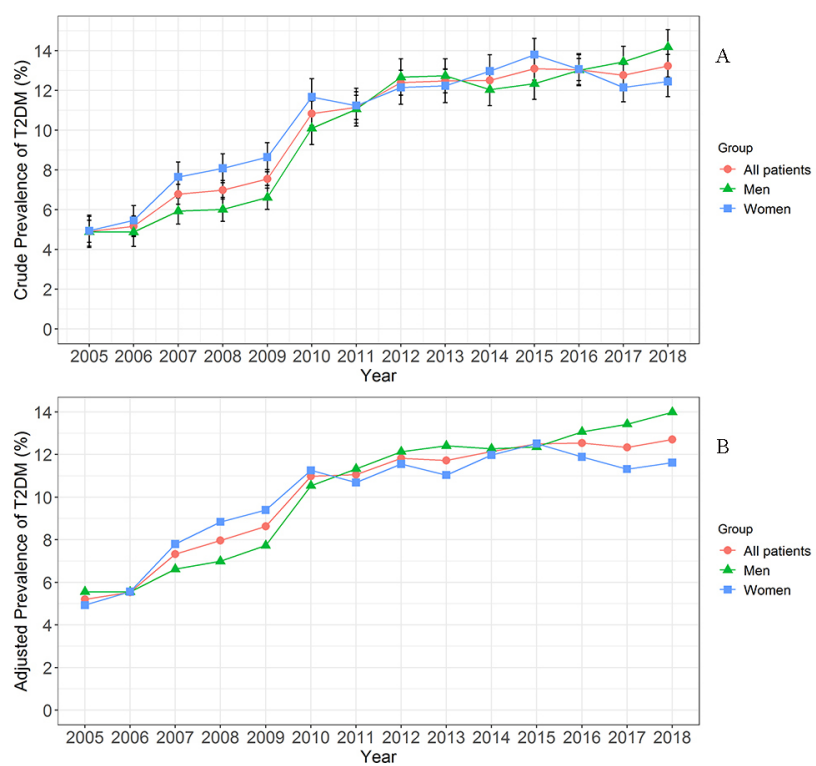

Figure 1 Trend in prevalence of T2DM in adult psychiatric inpatients during 2005-2018 in Beijing, China. (A) Crude prevalence of T2DM. (B) Adjusted prevalence of T2DM according to the overall participant population. T2DM, type 2 diabetes mellitus.

ethnic groups compared with Han participants, except for Manchu participants. In our study, the prevalence of diabetes among inpatients of other ethnicities was lower than that in Han inpatients, but no significant difference was observed after adjustment for other variables. The differences in T2DM prevalence between inpatients of Han ethnicity and those of other ethnicities might be related to substantial differences in genetic backgrounds, socioeconomic levels, lifestyle, and dietary patterns. ${ }^{5}$

Our study revealed that inpatients who had never been married had lower T2DM prevalence than married inpatients. A possible reason is that inpatients who had never been married tended to be younger. Meanwhile, inpatients who were widowed had higher prevalence for T2DM; this might be due to the lack of social support from a spouse. Previous studies have shown that adults

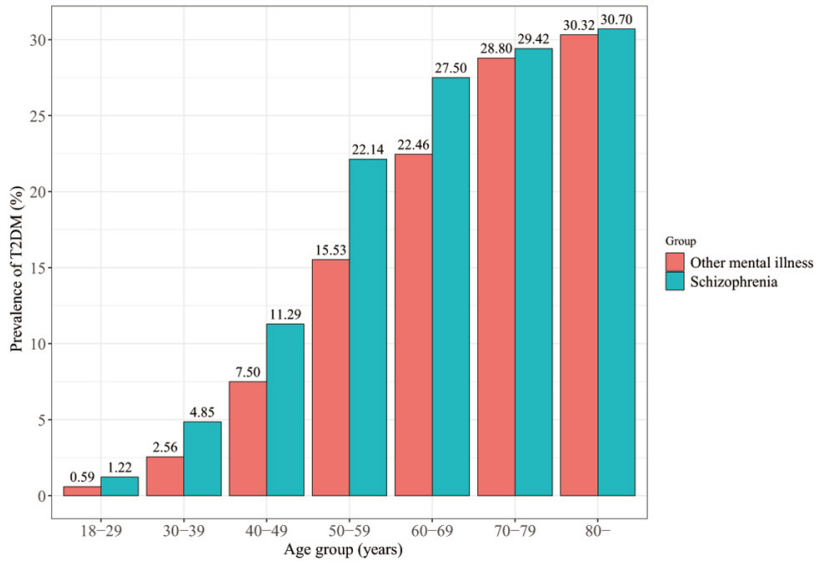

Figure 2 Age-specific prevalence of type 2 diabetes mellitus (T2DM) in inpatients with schizophrenia and other psychiatric disorders during 2005-2018 in Beijing, China. with SMI have inadequate knowledge of diabetes and have poor self-care practices, which may be a result of cognitive challenges, poor communication and compliance difficulties. ${ }^{20}$ Our results indicated that mental health providers play an integral role in the care of adult psychiatric inpatients, particularly elderly inpatients and those with limited family support. Furthermore, basic information regarding diabetes self-management and diabetes education should be provided to patients and their family members.

Our study also found that inpatients with UEBMI and URBMI payments exhibited higher prevalence of T2DM than inpatients with NCMS payments. UEBMI and URBMI are types of insurance for urban employees and residents, whereas NCMS is insurance for rural residents. A large prospective study on adults from urban and rural areas in China showed that diabetes prevalence was higher in urban areas than in rural areas $(8.1 \%$ vs $4.1 \%$, respectively). ${ }^{22}$ The differences in socioeconomic levels, lifestyle, and dietary patterns between urban and rural areas can possibly account for the difference in T2DM prevalence.

Compared with inpatients with other psychiatric disorders, such as bipolar affective disorder, recurrent depressive disorder, depressive episode, mental and behavioral disorders due to use of alcohol, and acute and transient psychotic disorders, those with schizophrenia had a higher prevalence of T2DM. This trend persisted even after adjustment for other variables; the aOR for inpatients with schizophrenia was 1.23 , which indicates that inpatients with schizophrenia have a higher probability of suffering from T2DM than those with other psychiatric disorders. After stratification by schizophrenia and other psychiatric disorders, we found that among young inpatients (18-39 years), those with schizophrenia exhibited higher diabetes prevalence than did those with other psychiatric disorders. This finding suggests that diabetes screening in inpatients with schizophrenia should begin at the earliest possible age.

Some antipsychotics, such as olanzapine and clozapine, have severe weight-gain side effects. ${ }^{23}$ A possible reason is that patients treated with these drugs may develop abnormal eating behaviors in response to altered appetite sensations and increased susceptibility to hunger, which may lead to a positive energy balance and contribute to gain in body weight, thus increasing the risk of T2DM. ${ }^{23}$ Besides, impaired glucose tolerance has been shown to occur even without weight gain in patients taking antipsychotics. ${ }^{23}$ In the present study, inpatients with antipsychotics use had significantly higher T2DM prevalence than those without antipsychotics use. However, the difference in T2DM prevalence became non-significant after adjustment for other variables. This result might be due to an underestimation of antipsychotics use in the inpatient population. In our study, $32.86 \%$ of inpatients received prescription of antipsychotic medication, according to the medical records. Inpatients who had a history of antipsychotic medication use might not 
have been identified in this study. Nevertheless, psychiatrists should, regardless of the medication prescribed, monitor and record waist circumference in every patient with schizophrenia at every visit, and should encourage patients to monitor and record their own weight. ${ }^{24}$

As T2DM, metabolic syndrome and non-alcoholic fatty liver disease are closely related, ${ }^{25}$ we included hypertension, lipid disorders, and fatty liver as covariates in this study. Patients with schizophrenia were reported to exhibit poor dietary habits, mainly characterized by a high intake of saturated fat and a low intake of fiber and fruit. ${ }^{26}$ Such a diet is more likely to increase the risk of metabolic abnormalities, including central obesity, hypertension, dyslipidemia, and glucose intolerance. ${ }^{27}$ Although hypertension and lipid disorders were not specific risk factors among psychiatric patients, these factors were more common in people with psychotic disorders due to adverse health behaviors, such as low levels of physical activity and poor diet. ${ }^{7}$ Foley and colleagues ${ }^{19}$ found that treated hypercholesterolemia and treated hypertension were strong predictors of T2DM, and the OR of DM development was 3.23 in psychiatric patients with hypertension. Our findings are consistent with those of Foley and colleagues ${ }^{19}$ study. In our study, the aORs were 3.09 in inpatients with hypertension and 1.95 in those with lipid disorders, which indicate that inpatients with hypertension or lipid disorders have a higher probability of suffering from T2DM than those without these conditions. Thus, risk factors such as hypertension and dyslipidemia established in the general population were also strongly associated with T2DM among psychiatric inpatients. Lowering blood pressure is the single most effective intervention for reducing cardiovascular morbidity and mortality in patients with T2DM in the general population. ${ }^{19}$ Our findings suggest that psychiatric inpatients with hypertension and lipid disorders have a higher prevalence of T2DM. Prevention and treatment of diabetes should be provided to these patients.

This study has several strengths. To our knowledge, this is by far the largest study on T2DM prevalence among adult psychiatric inpatients in China. We included all 19 specialized psychiatric hospitals in Beijing during 2005-2018. The prevalence and trend of T2DM among psychiatric inpatients in Beijing were accurately estimated. Furthermore, we used ICD-10 codes for disease diagnosis, which enables our results to be compared with those of similar studies.

This study has several limitations. First, our data covered all psychiatric inpatients from specialized psychiatric hospitals in Beijing and did not include patients in general hospitals and those who were not hospitalized. Thus, our results cannot be generalized to the entire population of patients with psychiatric disorders. Second, antipsychotics use in a population of inpatients might have been underestimated. Thus, additional studies on the association between antipsychotics use and T2DM in psychiatric inpatients are required. Finally, because we only included non-insulin-dependent patients with diabetes, individuals with T2DM who were dependent on insulin might be excluded; consequently, the prevalence of T2DM might have been underestimated.

In conclusion, the prevalence of T2DM was $10.75 \%$ in adult psychiatric inpatients in Beijing. Elderly inpatients as well as inpatients with schizophrenia, and those with hypertension, lipid disorders, and fatty liver exhibited higher T2DM prevalence. Psychiatric inpatients should be included in prevention strategies for diabetes, such as monitoring of metabolic conditions and lifestyle interventions, in combination with treatment for psychiatric disorders. Appropriate detection and treatment of abnormal glucose metabolism are crucial for interventions to improve clinical outcomes in psychiatric inpatients.

\section{Author affiliations}

${ }^{1}$ Beijing Huilongguan Hospital, Peking University Huilongguan Clinical Medical School, Beijing, China

${ }^{2}$ Department of Epidemiology and Biostatistics, Peking University School of Public Health, Beijing, China

${ }^{3}$ Beijing Municipal Commission of Health and Family Planning Information Center, Beijing Municipal Commission of Health and Family Planning Policy Research Center, Beijing, China

${ }^{4}$ Beijing Geriatric Hospital, Beijing, China

Acknowledgements We would like to thank all patients and health workers in 19 specialized psychiatric hospitals in Beijing. We also thank the Beijing Municipal Commission of Health and Family Planning Information Center for providing the data.

Contributors QM and FY searched the literature, designed the study, analyzed the data, interpreted the results, and drafted the manuscript. JLiu, BM, MG, FL, JLi and ZW collected the data and revised the manuscript. ML conceived, designed and supervised the study, interpreted the results, and revised the manuscript. All authors have approved the final version of the manuscript. ML is the guarantor.

Funding This work was supported by the National Natural Science Foundation of China (grant numbers 71874003 and 81703240). The funder had no role in study design, data collection, data analysis, data interpretation, or writing of the report. The corresponding author had access to all data in the study and had final responsibility for the decision to submit for publication.

Competing interests None declared.

Patient consent for publication Not required.

Ethics approval The study used information that is available in the database of the Beijing Municipal Commission of Health and Family Planning Information Center, and all identifiable information was removed.

Provenance and peer review Not commissioned; externally peer reviewed.

Data availability statement Data may be obtained from a third party and are not publicly available.

Open access This is an open access article distributed in accordance with the Creative Commons Attribution Non Commercial (CC BY-NC 4.0) license, which permits others to distribute, remix, adapt, build upon this work non-commercially, and license their derivative works on different terms, provided the original work is properly cited, appropriate credit is given, any changes made indicated, and the use is non-commercial. See: http://creativecommons.org/licenses/by-nc/4.0/.

ORCID ID

Min Liu http://orcid.org/0000-0002-5059-3743

\section{REFERENCES}

1 Hu FB. Globalization of diabetes: the role of diet, lifestyle, and genes. Diabetes Care 2011;34:1249-57.

2 DeFronzo RA, Ferrannini E, Groop L, et al. Type 2 diabetes mellitus. Nat Rev Dis Primers 2015;1:15019. 
3 International Diabetes Federation. IDF diabetes atlas. 8th edn, 2017. https://diabetesatlas.org/IDF_Diabetes_Atlas_8e_interactive_EN/

4 Zhang N, Du SM, Ma GS. Current lifestyle factors that increase risk of T2DM in China. Eur J Clin Nutr 2017;71:832-8.

5 Wang L, Gao P, Zhang M, et al. Prevalence and ethnic pattern of diabetes and prediabetes in China in 2013. JAMA 2017;317:2515-23.

6 Roberts $\mathrm{E}$, Jones L, Blackman A, et al. The prevalence of diabetes mellitus and abnormal glucose metabolism in the inpatient psychiatric setting: a systematic review and meta-analysis. Gen Hosp Psychiatry 2017;45:76-84.

7 Ward M, Druss B. The epidemiology of diabetes in psychotic disorders. Lancet Psychiatry 2015;2:431-51.

8 Sun L, Getz M, Daboul S, et al. Independence of diabetes and obesity in adults with serious mental illness: findings from a large urban public hospital. J Psychiatr Res 2018;99:159-66.

9 Vancampfort D, Correll CU, Galling B, et al. Diabetes mellitus in people with schizophrenia, bipolar disorder and major depressive disorder: a systematic review and large scale meta-analysis. World Psychiatry 2016;15:166-74

10 Correll CU, Joffe BI, Rosen LM, et al. Cardiovascular and cerebrovascular risk factors and events associated with secondgeneration antipsychotic compared to antidepressant use in a nonelderly adult sample: results from a claims-based inception cohort study. World Psychiatry 2015;14:56-63.

11 DE Hert M, Correll CU, Bobes J, et al. Physical illness in patients with severe mental disorders. I. prevalence, impact of medications and disparities in health care. World Psychiatry 2011;10:52-77.

$12 \mathrm{Liu} \mathrm{JH}$, Zhao N. Analysis of occurrence of diabetes of inpatients with schizophrenia and relevant factors. China Foreign Medical Treatment 2017:36:106-8.

13 Ban CX, Zhang QY, HJ L, et al. Analysis of contributing factors for somatic diseases in hospitalized elderly schizophrenics. Chinese Journal of Geriatrics 2018;37:1243-7.

14 World Health Organization. International statistical classification of diseases and health related problems. 10th edn. Beijing: People's Medical Publishing House, 1996.

15 Zou X, Zhou X, Zhu Z, et al. Novel subgroups of patients with adultonset diabetes in Chinese and US populations. Lancet Diabetes Endocrinol 2019;7:9-11.
16 Ahlqvist E, Storm P, Käräjämäki $A$, et al. Novel subgroups of adult-onset diabetes and their association with outcomes: a datadriven cluster analysis of six variables. Lancet Diabetes Endocrinol 2018:6:361-9.

17 Crump C, Winkleby MA, Sundquist K, et al. Comorbidities and mortality in persons with schizophrenia: a Swedish national cohort study. Am J Psychiatry 2013;170:324-33.

18 Das-Munshi J, Ashworth M, Dewey ME, et al. Type 2 diabetes mellitus in people with severe mental illness: inequalities by ethnicity and age. cross-sectional analysis of 588408 records from the UK. Diabet Med 2017;34:916-24.

19 Foley DL, Mackinnon A, Morgan VA, et al. Predictors of type 2 diabetes in a nationally representative sample of adults with psychosis. World Psychiatry 2014;13:176-83.

20 Wykes TL, Lee AA, Bourassa K, et al. Diabetes knowledge among adults with serious mental illness and comorbid diabetes mellitus. Arch Psychiatr Nurs 2017;31:190-6.

21 Grøn AO, Dalsgaard E-M, Ribe AR, et al. Improving diabetes care among patients with severe mental illness: a systematic review of the effect of interventions. Prim Care Diabetes 2018;12:289-304

22 Bragg F, Holmes MV, lona A, et al. Association between diabetes and cause-specific mortality in rural and urban areas of China. JAMA 2017;317:280-9.

23 Deng C. Effects of antipsychotic medications on appetite, weight, and insulin resistance. Endocrinol Metab Clin North Am 2013;42:545-63.

24 Vancampfort $\mathrm{D}$, Wampers $\mathrm{M}$, Mitchell AJ, et al. A meta-analysis of cardio-metabolic abnormalities in drug naïve, first-episode and multi-episode patients with schizophrenia versus general population controls. World Psychiatry 2013;12:240-50.

25 Firneisz $\mathrm{G}$. Non-Alcoholic fatty liver disease and type 2 diabetes mellitus: the liver disease of our age? World $\mathrm{J}$ Gastroenterol 2014;20:9072-89.

26 Dipasquale S, Pariante CM, Dazzan P, et al. The dietary pattern of patients with schizophrenia: a systematic review. J Psychiatr Res 2013;47:197-207.

27 Papanastasiou E. The prevalence and mechanisms of metabolic syndrome in schizophrenia: a review. Ther Adv Psychopharmacol 2013;3:33-51. 